\title{
Genetic and chemical inhibition of autophagy in zebrafish induced myeloproliferation
}

Kazi Md Mahmudul Hasan*1, Xiang-Ke Chen*1,2 ${ }^{*}$ Zhen-Ni Yi ${ }^{1}$, Jack Jark-Yin Lau ${ }^{1}$ and Alvin Chun-Hang $\mathrm{Ma}^{1}$

${ }^{1}$ Department of Health Technology and Informatics, The Hong Kong Polytechnic University, Hong Kong, China;

${ }^{2}$ Laboratory of Neurodegenerative Diseases, School of Biomedical Sciences, The University of Hong Kong, Pokfulam, Hong Kong, China;

\section{Authors' contribution:}

$\mathrm{KMMH}$ and $\mathrm{XC}$ are co-first authors. KMMH performed most of the experiments and written the manuscript; XC contributed to experimental design, performed part of the experiment and written the manuscript; ZY and JJL performed part of the experiment; ACM conceptualized, designed, supervised the whole project and written the manuscript

\section{Running heads:}

Autophagy deficiency induced myeloproliferation

\section{Corresponding author:}

Alvin Chun-Hang Ma

alvin.ma@polyu.edu.hk

\section{Word count:}

Abstract: 203; Main text: 2,922

\section{Acknowledgments.}

Zebrafish maintenance was supported by Fish Model Translational Research Laboratory (HTI, PolyU). Microscopic imaging was supported by University Research Facility in Life Sciences (ULS, PolyU). Transgenic zebrafish lines $\operatorname{Tg}(G F P-L c 3)$ and $\operatorname{Tg}($ corola:mCherry) were kindly provided by Dr. X Xu (Mayo Clinic, Rochester, MN) and Prof. Z Wen (HKUST, HK, China), 
bioRxiv preprint doi: https://doi.org/10.1101/2021.06.14.448302; this version posted June 14, 2021. The copyright holder for this preprint (which was not certified by peer review) is the author/funder, who has granted bioRxiv a license to display the preprint in perpetuity. It is made available under aCC-BY-NC-ND 4.0 International license.

respectively. This work is supported by the FHB HMRF [03143765] and FHB HMRF [06173226] to ACM. 


\begin{abstract}
Autophagy is an evolutionary conserved and dynamic lysosomal degradation process for cellular homeostasis and remodelling, which is essential for the development and maintenance of different hematopoietic fates. However, the roles of autophagy in definitive hematopoiesis remain elusive. Here, we exploited zebrafish (Danio rerio) to investigate the effect of knocking-out unc51 like autophagy activating kinase $1 \mathrm{~b}$ and 2 ( $u l k 1 b$ and $u l k 2)$, homologous of human ULK1 and ULK2, respectively, on definitive hematopoiesis. Upon $u l k 1 b$ or $u l k 2$ mutation, autophagosome formation was blocked in zebrafish embryos. More importantly, pan-leukocytes (lcpl), common myeloid progenitors (spilb), neutrophils ( $m p x$ ), and macrophages (mpeg1.1) significantly elevated, while the hematopoietic stem and progenitor cell (HSPC) (myb), erythroid progenitors (gatal), and embryonic hemoglobin (hbael.1) significantly reduced in the caudal hematopoietic tissue (CHT) of $u l k 1 b$ or $u l k 2$ mutant zebrafish embryos. On the other hand, chemically modulated autophagy induction by calpeptin, a downstream autophagy inducer for $u l k$ complex, was insufficient to ameliorate dysregulated hematopoiesis in both $u l k 1 b$ and $u l k 2$ mutants. Conversely, autophagy inhibitor 3-Methyladenine functioned parallelly with the ulk mutants to maintain defective hematopoiesis. These observations raised a link between autophagy and definitive hematopoiesis and potentiates the fact that autophagy deficiency incorporates with myeloproliferation and anemia, which warrants the significance of autophagy in regulating definitive hematopoiesis.
\end{abstract}




\section{Introduction}

Macroautophagy/autophagy is an adaptive and highly conserved metabolic process characterized by double membrane vesicle formation (autophagosome) and lysosome-dependent degradation under various physiological and pathophysiological conditions such as cellular development and differentiation, immunity, cancer, and aging. ${ }^{1-4}$ Autophagy is initiated with the activation of unc51 like autophagy activating kinase (ULK1) complex, which primarily consists of ULK1, autophagy-related protein 13 (ATG13), focal adhesion kinase family-interacting protein of 200 kDa (FIP200), ATG101, ULK2 and subsequently regulated by a series of ATGs, such as ATG5, Beclin $1(\mathrm{BECN})$, and ATG7. ${ }^{5}$ In particular, both ULK1 and ULK2 have redundant roles in the canonical autophagy machinery and double knockout of ULK1/2 in mice showed neonatal mortality ${ }^{6}$ similar to the loss of other core autophagy genes such as ATG5, ${ }^{7}$ ATG7, ${ }^{8}$ ATG9a ${ }^{9}$ and ATG16L. ${ }^{10}$ As a kinase protein of the ULK1 complex, the ULK1 initiates the phosphorylation of Beclin-1 and vacuolar protein sorting 34 (VPS34) lipid kinase, ${ }^{11}$ ATG14 ${ }^{12}$ and promotes membrane recycling via ATG9. ${ }^{13,14}$ Moreover, the ULK1-FIP200 protein complex plays crucial role during the formation of starvation-induced ATG8/LC3 (microtubule-associated protein light chain 3) puncta and omegasomes on which phagophores emerge. ${ }^{15}$ In addition to canonical autophagy, non-canonical autophagy was also identified, which involves only a subset of ATGs. ${ }^{16}$ For instance, ULK1-dependent ATG5-independent non-canonical autophagy is involved in the clearance of mitochondria from reticulocytes. ${ }^{17}$ Besides, the LC3-associated phagocytosis (LAP), which is a non-canonical autophagy in phagocytes characterized by single-membrane vesicle formation, is ULK1-indepndent. ${ }^{18}$

The discovery of multiple autophagy-related genes (Atgs) in the past two decades has geared up research to understand the link between autophagy and hematopoiesis. Previous studies demonstrated that the loss of Atg7 in the mice hematopoietic systems leading to aberrant HSC functions, severe myeloproliferation, ${ }^{19,}{ }^{20}$ anemia and lymphopenia, ${ }^{21}$ while as a mammalian counterpart of yeast Atg17, conditional deletion of FIP200 in mice hematopoietic tissues resulted in HSC depletion, aberrant myeloid expansion and erythroblastic anemia. ${ }^{22}$ Thus, different ATGs may play distinct roles in hematopoiesis. In addition to normal hematopoiesis, autophagy also implicated in hematological malignancies. Similar to other cancers, ${ }^{23,24}$ the role of autophagy in hematological malignancies is paradoxical and could be either pro-oncogenic or anti-oncogenic 
depending on cellular context. ${ }^{25-28}$ Nevertheless, the precise role of ULK1 and ULK2 dependent autophagy in the context of hematopoiesis remains unclear. In particular, the complex network and underlying mechanism of canonical and non-canonical autophagy in regulating hematopoiesis remains to be investigated.

Zebrafish (Danio rerio) has emerged in recent years as a robust in vivo model for autophagy and hematopoietic research with unique characteristics, including high fecundity, optical transparency, and feasibility for genetic and chemical manipulation. The small vertebrate has been widely used to study hematopoiesis and model human hematopoietic malignancies. ${ }^{29-31}$ While it remains difficult to experimentally observed autophagy in other whole-animal model organisms, zebrafish has been demonstrated as a unique vertebrate model to study autophagy in vivo at cellular level, ${ }^{32}$ highlighted the potential of using zebrafish model to study the complex role of autophagy in hematopoiesis. Here we used zebrafish $u l k 1 b$ and $u l k 2$ mutant models as the paradigm of autophagy dysfunction and depicted the previously unknown role of ulk-dependent autophagy in regulating definitive myelopoiesis.

\section{Methods}

\section{Zebrafish husbandry and maintenance}

Wild-type, $\operatorname{Tg}($ GFP-Lc3), and $\operatorname{Tg}($ corola:DsRed $)$ zebrafish lines were maintained under standard aquatic conditions. Fish was fed with hatched brine shrimp twice a day and embryos were collected from natural spawning and staged as described previously. ${ }^{33,34}$ Protocols for microinjection and whole-mount in situ hybridization (WISH) have been described previously. ${ }^{29,}{ }^{35}$ Conventional pigmentation inhibition with 1-phenyl 2-thiourea (PTU) was refrained to avoid unwanted autophagy induction. ${ }^{32}$ Fixed embryos were bleached with $1 \% \mathrm{KOH}$ and $3 \% \mathrm{H}_{2} \mathrm{O}_{2}$ before WISH. Live imaging was performed using Lightsheet fluorescent microscope. All animal experiments were conducted in accordance with protocols approved by the Animal Subjects Ethics SubCommittee (ASESC) of The Hong Kong Polytechnic University.

\section{Generation of the $u l k 1 a, u l k 1 b$ and $u l k 2$ mutants by TALEN}

Transcription activator-like effector nucleases (TALEN) pairs targeting genomic sequence of zebrafish ulkla Exon-2, ulk1b Exon-4 and ulk2 Exon-7 were designed and synthesized as described previosuly. ${ }^{36,37}$ mRNA encoding $u l k l a, u l k 1 b$ and $u l k 2$ TALEN pairs were in vitro transcribed 
with T3 mMessage mMachine transcription Kit (Ambion, \#AM1348) and microinjected into the yolk of one-cell-stage zebrafish embryos. Mutagenic activities were confirmed by restriction fragment length polymorphism (RFLP) assay and Sanger sequencing as previously described. ${ }^{38}$ Stable $u l k 1 b$ mutants $\left(u l k 1 b^{--}\right)$and $u l k 2$ mutants $\left(u l k 2^{-/-}\right)$were obtained from heterozygous $u l k 1 b$ mutants $\left(u l k 1 b^{+/-}\right)$and $u l k 2$ mutants $\left(u l k 2^{+/-}\right)$incross respectively. We further incrossed the $u l k 1 b^{+/-}$ mutants with the double transgenic $\operatorname{Tg}(G F P-L c 3$;corola:DsRed $)$ to obtain the heterozygous mutants and subsequently incross the heterozygous mutants to obtain the double transgenic homozygous ulk1b mutants [Tg(ulk1 ${ }^{-/}:$GFP-Lc3;corola:DsRed $\left.)\right]$.

\section{Western blot analysis}

Embryos were deyolked before mechanically homogenized in CelLytic ${ }^{\mathrm{TM}}$ MT Cell Lysis Reagent (Sigma Aldrich, \#C3228). Protein concentrations were measured by BCA assay kits (Thermo Scientific ${ }^{\mathrm{TM}}, \# 23225$ ) and protein lysates were mixed with sodium dodecyl sulfate (SDS) loading buffer and heat denatured. Protein samples were then electrophoresed on $12 \% \mathrm{TGX}^{\mathrm{TM}}$ FastCast ${ }^{\mathrm{TM}}$ Acrylamide Kit (Bio-Rad Laboratories, Inc., \#1610175), transferred to polyvinylidene difluoride (PVDF) membrane (Bio-Rad Laboratories, \#1620264) and blocked in 5\% non-fat dry milk (BioRad Laboratories, \#1706404). Membranes were then hybridized with anti-Lc3b (Abcam, \#ab48394; 1:1000) or anti-GAPDH (Cell Signaling Technology, \#2118; 1:20000) at $4^{0} \mathrm{C}$ overnight. PVDF membranes were further washed in TBST and incubated with goat anti-rabbit secondary antibody (Abcam, \#ab6721; 1:3000) for 2 hours at room temperature before signal development with Western ECL Substrate (Bio-Rad Laboratories, \#1705061) and imaging under ChemiDoc XRS+ System (Bio-Rad Laboratories).

\section{Autophagy modulator treatment and LysoTracker probe staining}

Embryos were treated with calpeptin (Selleckchem, \#S7396), chloroquine (Selleckchem, \#S4157) and 3-Methyladenine (3- MA) (Selleckchem, \#S2767) at 50 $\mu \mathrm{M}, 100 \mu \mathrm{M}$ and $10 \mathrm{mM}$, respectively. Fluorescent dye LysoTrackerTM Red DND-99 (Invitrogen, \#L7528) targeting lysosomes was diluted to a final concentration of $10 \mu \mathrm{M}$. $4 \mathrm{dpf} \operatorname{Tg}(G F P-L c 3)$ zebrafish embryos were incubated with the diluted LysoTrackerTM Red DND-99 in dark at $28.5^{\circ} \mathrm{C}$ for 45 minutes. ${ }^{39,40}$ Subsequently, embryos were rinsed 3 times with E3 fish water prior to imaging.

\section{Lightsheet and confocal microscopic imaging}


Zebrafish embryos were anesthetized with tricaine (Sigma-Aldrich, \# A5040) at a concentration of $0.164 \mathrm{mg} / \mathrm{ml}$ and mounted in $1.5 \%$ low gelling temperature agarose (Sigma-Aldrich, \# A9045) into glass capillary and $35 \mathrm{~mm}$ glass-bottom confocal dish for light sheet and confocal imaging, respectively. Live images were acquired by Zeiss Lightsheet Z.1 Selective Plane Illumination Microscope (Carl Zeiss Microscopy, NY, USA) with a 20x objective lens and Leica TCS SPE Confocal Microscope (Leica Microsystems, Wetzlar, Germany) with the 10x and 40x objective lenses. Images were further processed and analyzed with ZEN (Carl Zeiss Microscopy, NY, USA), Leica LAS-X (Leica Microsystems, Wetzlar, Germany) imaging software and ImageJ (National Institutes of Health, USA), respectively.

\section{Phospho-Histone H3 (pH3) immunostaining}

$\operatorname{Tg}(m p x: E G F P)$ embryos at $3 \mathrm{dpf}$ were fixed with $4 \%$ paraformaldehyde (PFA) at room temperature for 4 hours and subsequently permeabilized with pre-chilled acetone at $-20{ }^{0} \mathrm{C}$ for 20 minutes. After washing with phosphate-buffered Saline with $0.1 \%$ Tween 20 Detergent (PBST), embryos were incubated in block buffer $(0.1 \%$ bovine serum albumin, $0.1 \%$ dimethyl sulfoxide (DMSO), 2\% normal goat serum and 0.2\% Triton-X100 in PBS) for 30 minutes and hybridized with polyclonal rabbit anti-phospho-Histone H3 (Ser10) antibody (Cell Signaling Technology, \#9701; 1:1000) at overnight at $4^{0} \mathrm{C}$. After washing with PBST, embryos were incubated with Alexa Fluor 594 goat anti-rabbit secondary antibody (Invitrogen, \#A-11012) at 1:500 for an hour at room temperature before final PBST washing prior to imaging.

\section{Quantitative analysis and statistics}

To measure the relative number of autophagosome $\left(G F P-L c 3^{+}\right)$, lysosome $\left(\right.$LysoTracker $\left.\operatorname{Red}^{+}\right)$ and autolysosome $\left(G F P-L c 3^{+}\right.$and LysoTracker $\left.\operatorname{Red}^{+}\right)$puncta, maximum intensity projections (MIPs) were performed using Z-Stack images at the midbrain sections to monitor and quantify the relative autophagosome, lysosome and autolysosome numbers inside the neurons (20 out of 100 layers were taken to clearly monitor puncta per neuron). EGFP (green color) positive autophagosome puncta, mCherry (magenta color) positive lysosome puncta and white color positive autolysosome puncta were defined by fluorescent intensity occupying more than one pixel and distinguishable from the background signals. ${ }^{41}$ Overall puncta inside the neuron cells were counted from the whole midbrain section for each sample. The number of autophagosome, lysosome and autolysosome puncta per neuron cell was calculated by counting the total number of 
color specific puncta divided by the number of neuron cells in each embryo and at least 10 embryos were recruited to complete the triplicate. ImageJ software version 1.8.0 (NIH) was used to quantify western-blot results. Statistical analysis was performed by two-way analysis of variance (ANOVA) with Tukey's multiple comparisons tests in the mutant and drug treated groups comparing each cell mean with every other cell mean using GraphPad Prism, version 7 (GraphPad Software, CA, USA). Mann-Whitney nonparametric U-test were applied in other analysis. In all experiments, results were presented as mean \pm standard error of the mean (SEM) and P-value less than $0.05(\mathrm{P}<0.05)$ were considered statistically significant.

\section{Results}

\section{TALEN-mediated mutagenesis of zebrafish $u l k 1 b$ and $u l k 2$}

In zebrafish, $u l k l$ is duplicated into $u l k l a$ and $u l k l b$. Phylogenetic analysis revealed that zebrafish $u l k 1 b$ and $u l k 2$ are orthologues of human $U L K 1$ and $U L K 2^{42}$ (Figure S1A) with highly conserved syntenic region (Figure S1B). Zebrafish $u l k 1 b$ and $u l k 2$ expressed ubiquitously during early embryonic development and later predominantly in the head region (Figure S1C and S1D). While $u l k 1 b$ also expressed in somites at 24 hour-post-fertilization (hpf) (Figure S1C), both $u l k 1 b$ and $u l k 2$ expressed in trunk neural crest cells and caudal hematopoietic tissue (CHT) at 48 hpf (Figure $\mathrm{S} 1 \mathrm{C}$ and S1D). To investigate the role of autophagy in hematopoiesis, we targeted zebrafish $u l k 1 b$ and $u l k 2$ with TALEN (Figure S2A and S2B) and somatic targeting of ulklb Exon-4 and ulk2 Exon-7 were confirmed by RFLP assay (Figure S2C). Stable heterozygous F1 carried a 5-base pair (bp) and 7-bp frame-shifting deletion in $u l k 1 b$ and $u l k 2$, respectively, were identified and confirmed with Sanger sequencing, which will result in pre-mature stop and truncation of ulk1b and ulk2 (Figure S2D and S2E).

\section{Autophagy activation, but not autophagy flux was suppressed in $u l k 1 b$ and $u l k 2$ mutants}

We first examined autophagy in $u l k 1 b$ mutant. Stable homozygous $u l k 1 b$ mutants ( $u l k 1 b^{-/}$) embryos with $\operatorname{Tg}(G F P-L c 3)$ background were stained with Lysotracker Red and the relative number of autophagosomes, lysosomes and autolysosomes were significantly decreased in $u l k 1 b^{-}$ / mutants (Figure 1A), indicated a decrease in autophagy activation. Similar results were observed in somatic $u l k 2$ mutant $\left(u l k 2^{M u t}\right.$ ) though lysosome numbers were not notably affected. (Figure S3A). Western blot also confirmed the decrease in Lc3-II/GAPDH (Figure 1B and S3B). 
Concomitant treatments with late-stage autophagy inhibitor, chloroquine (CQ) was performed to examine autophagy flux. Albeit the relative number of autophagosomes were significantly decreased in both $u l k 1 b^{-/ t}$ and $u l k 2^{M u t}$, the fold-increase in autophagosome numbers after CQ treatment was comparable between $u l k$ mutants and control, indicated that the autophagy flux was not perturbed in $u l k 1 b^{-/-}$and $u l k 2^{M u t}$ mutants (Figure $1 \mathrm{~A}$ and S3A). Autophagy in leukocytes were also examined using $\operatorname{Tg}($ GFP-Lc3;corola:DsRed $)$ double transgenic line. Similar to nonhematopoietic tissues, the relative number of $l c 3$-postive puncta (autophagosomes) in corolapositive cells also decreased in $u l k 1 b^{-/}$and $u l k 2^{M u t}$ (Figure $1 \mathrm{C}$ and S3C).

\section{$u l k 1 b$ and $u l k 2$ deficiency induced myeloproliferation}

The effects of $u l k 1 b$ and $u l k 2$ deficiency on definitive hematopoiesis were examined by wholemount in situ hybridization (WISH). Increased expression of makers associated with myeloid lineages, including myeloid progenitor $(s p i l b)$, pan-leukocyte (lcpl) and neutrophil (mpx) were

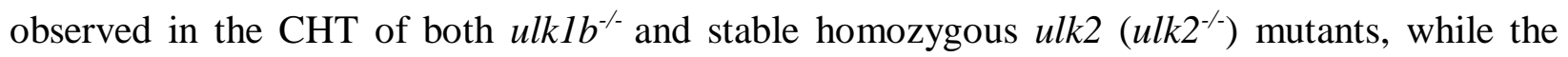
expression of markers associated with hematopoietic stem and progenitors (HSPCs) (myb) and erythroids (hbae1.1) were significantly decreased (Figure 2A and 2B), suggested that $u l k 1 b$ and ulk2 knocking-out perturbed definitive hematopoiesis, in particular, induced myeloproliferation.

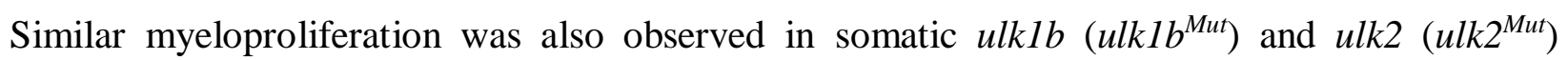
mutants (Figure S4A and S4B). Proliferation in neutrophils was examined by immunostaining of phosphohistone $\mathrm{H} 3(\mathrm{pH} 3)$ in $\mathrm{Tg}(m p x: E G F P)$ at $72 \mathrm{hpf}$. In both $u l k 1 b^{M u t}$ and $u l k 2^{M u t}$, the percentage of pH3-postive neutrophils significantly increased (Figure S5).

\section{Treatment with autophagy modulators also perturbed definitive hematopoiesis}

To examine if the hematopoietic phenotypes observed in $u l k 1 b^{-/}$and $u l k 2^{-/-}$were autophagydependent, treatment with autophagy modulators, including 3-MA and calpeptin (autophagy inducer) were performed. Autophagy activation was significantly suppressed in embryos treated with 3-MA as shown by the decreased number of autophagosomes and autolysosomes (Figure 3A). Similar to $u l k 1 b$ and $u l k 2$ mutants, treatment with 3-MA also induced myeloproliferation as shown by the increase in lcpl and $m p x$ (Figure 3B). Expression of $m y b$ were markedly decreased while hhabe1.1 were unaffected (Figure 3B). In contrary, calpeptin-treatment significantly increased the number of autophagosomes and autolysosomes in zebrafish embryos (Figure 4A). Also opposite to 3-MA treatment, calpeptin significantly decreased the expression of $l c p l$ and $m p x$, while the 
expression of myb and hbael.1 were significantly increased (Figure 4B and Figure S6). More importantly, both $u l k 1 b$ and $u l k 2$ knock-out completely reverted the effects of calpeptin treatment on autophagy and hematopoiesis (Figure 4B and Figure S6).

\section{Discussion}

In this study, we took advantages of the optically clear and robust zebrafish model to investigate the role of autophagy in hematopoiesis. To model defective autophagy, we genetically targeted zebrafish $u l k 1 b$ and $u l k 2$ with TALEN as described previously. ${ }^{43}$ Zebrafish $u l k 1$ is duplicated into $u l k 1 a$ and $u l k 1 b$ and phylogenetic analysis revealed that $u l k 1 b$ is the orthologous of human ULK1 and ulkla knockout did not significantly affect autophagy in zebrafish embryos (data not shown). Both $u l k 1 b$ and $u l k 2$ expressed ubiquitously during early embryonic development and later predominantly in the head region but also in neural crest cells, somites and CHT. The expression pattern highlighted the importance of autophagy during early embryogenesis as well as the development of neural crest cells, and explained the high autophagy level observed in skin, eye, brain and muscle cells. ${ }^{32}$ Both somatic $u l k 1 b^{M u t}$ and $u l k 2^{M u t}$ as well as homozygous $u l k 1 b^{-/-}$and $u l k 2^{--}$mutants survived with normal gross development probably due to the functional redundancy between Ulk1 and Ulk2. ${ }^{44}$

Defective autophagy was confirmed in both stable homozygous $u l k 1 b^{-/}$and somatic $u l k 2^{M u t}$ mutants by the significantly decreased number of autophagosome and autolysosome. Treatment with CQ, which blocks the fusion of autophagsome and lysosome in the late stage of autophagy ${ }^{45}$, did not rescued the decrease in autophagosome and autolysosome numbers observed in $u l k 1 b^{-/}$ and $u l k 2^{M u t}$, suggested the blockage in autophagosome formation during early autophagy activation. While early autophagy activation was suppressed, autophagy flux was not significantly affected, which could be explained by the function of ULK1 complex in autophagy activation. ${ }^{46}$ Consistent with the expression of $u l k 1 b$ and $u l k 2$ in CHT, autophagy puncta was also detected in corola-positive myeloid cells and more importantly, significantly decreased upon genetic targeting of $u l k 1 b$ and $u l k 2$, suggested that autophagy involves in definitive hematopoiesis, particularly in myelopoiesis. 
We then examined the effects of $u l k 1 b$ and $u l k 2$ mutations in definitive hematopoiesis by WISH. In particular, expansion in myeloid lineages in the expenses of HSPC and erythroid lineages were observed in homozygous mutants of $u l k 1 b$ and $u l k 2$, demonstrated the role of Ulk1 complex in regulating definitive hematopoiesis. Interestingly, aberrant proliferation in mpx-expressing neutrophils was also observed in ulk mutant models, which is similar to the phenotypes in Atg5 knock-out mice previously reported with increased and rapid production of neutrophils ${ }^{47}$ and suggested Ulk and Atg5-dependent autophagy may limit immature neutrophil. To investigate if the hematopoietic phenotypes observed in $u l k 1 b$ and $u l k 2$ mutants were autophagy-dependent, we chemically modulated autophagy with 3-MA and calpeptin. While treatment with 3-MA, which is a PI3K inhibitor blocking early autophagosome formation, recapitulated the myeloproliferation and treatment with autophagy inducer, calpeptin, suppressed myeloid lineages. In particular, genetic targeting of $u l k 1 b$ and $u l k 2$ completely abrogated the effects of calpeptin treatment on autophagy and myeloid lineages, suggested that the ulk-deficiency induced myeloproliferation is autophagy-dependent. Nevertheless, further investigation is needed to confirm if Ulks exhibit noncanonical or autophagy-independent regulation on hematopoiesis.

During selective and nonselective autophagy, both ULK1 and ULK2 play critical roles in response to mitochondrial damage, infections and metabolic stresses in mammalian cells. ${ }^{48,49}$ Likely to the ATG5 and ATG7 deficient in vivo mice models of hematopoietic systems, where all of which mediate the depletion of autophagosome or Lc3-IIB puncta in the hematopoietic tissue under basal physiologic conditions, ${ }^{50,51}$ here, upon genetic and chemical targeting of autophagy, we demonstrated that $u l k l b$ and $u l k 2$ are also important to maintain "basal" autophagy and normal hematopoiesis in zebrafish embryos. Interestingly, hematopoietic ablation of $U l k 1$ in $\beta$-thalassemic mice exacerbates disease phenotypes whereas Atg5 knockout has comparatively minor effects indicating a Ulk1 dependent and Atg5 independent role during hematopoiesis. ${ }^{52}$ Nevertheless, further targeting multiple and major autophagy genes either alone or in combination, downstream to the ULK1 complex, regulating different steps of the canonical pathway would further clarify the precise roles of ULK1 and ULK2 in hematopoiesis. 
Autophagy has been reported to implicate in hematological malignancies. While many studies have reported that autophagy inhibition overcomes drug resistance ${ }^{53,54}$ and suppresses leukemia cell growth, ${ }^{5,56}$ others have demonstrated that autophagy suppression is important for leukemia development. ${ }^{57,58}$ These contradictive results suggested that autophagy plays paradoxical roles in leukemogenesis depending on cellular contexts. Our results showing that autophagy suppression would lead to deregulation of normal myelopoiesis, demonstrating the important anti-oncogenic role of autophagy during early stage of myeloid malignancies. Further investigation with bona fide models of hematological malignancy and tissue specific knock-out of autophagy from the myeloid cell are warranted. For instance, zebrafish has emerged as an important model organism for human cancer and models of hematological malignancies including myeloproliferative neoplasm (MPN), acute myeloid leukemia (AML), chronic myeloid leukemia (CML) and acute lymphoblastic leukemia (ALL) with highly conserved oncogenic pathways and pharmacologic responses were reported. ${ }^{59-63}$ With the well-developed methodologies in autophagy study ${ }^{32,39,64}$ and genetic engineering, ${ }^{65}$ zebrafish could be developed into a unique modelling platform to define the role of autophagy in hematological malignancies, which will provide important information for the development of autophagy-related therapeutic strategies against these heterogeneous diseases.

\section{References}

1. Mizushima N, Levine B. Autophagy in mammalian development and differentiation. Nature cell biology 2010, 12(9):823-30.

2. Zhou X-J, Zhang H. Autophagy in immunity: implications in etiology of autoimmune/autoinflammatory diseases. Autophagy 2012, 8(9):1286-1299.

3. White E. The role for autophagy in cancer. The Journal of clinical investigation 2015, 125(1):42-46.

4. Rubinsztein David C, Mariño G, Kroemer G. Autophagy and Aging. Cell 2011, 146(5):682695.

5. Ganley IG, Lam DH, Wang J, Ding X, et al. ULK1.ATG13.FIP200 complex mediates mTOR signaling and is essential for autophagy. J Biol Chem 2009, 284(18):12297-12305.

6. Cheong H, Lindsten T, Wu J, Lu C, et al. Ammonia-induced autophagy is independent of ULK1/ULK2 kinases. Proceedings of the National Academy of Sciences 2011, 108(27):1112111126.

7. Yoshii Saori R, Kuma A, Akashi T, Hara T, et al. Systemic Analysis of Atg5-Null Mice Rescued from Neonatal Lethality by Transgenic ATG5 Expression in Neurons. Developmental cell 2016, 39(1):116-130. 
8. Komatsu M, Waguri S, Ueno T, Iwata J, et al. Impairment of starvation-induced and constitutive autophagy in Atg7-deficient mice. The Journal of cell biology 2005, 169(3):425-34.

9. Saitoh T, Fujita N, Hayashi T, Takahara K, et al. Atg9a controls dsDNA-driven dynamic translocation of STING and the innate immune response. Proceedings of the National Academy of Sciences of the United States of America 2009, 106(49):20842-6.

10. Saitoh T, Fujita N, Jang MH, Uematsu S, et al. Loss of the autophagy protein Atg16L1 enhances endotoxin-induced IL-1beta production. Nature 2008, 456(7219):264-8.

11. Russell RC, Tian Y, Yuan H, Park HW, et al. ULK1 induces autophagy by phosphorylating Beclin-1 and activating VPS34 lipid kinase. Nature cell biology 2013, 15(7):741-750.

12. Park JM, Jung CH, Seo M, Otto NM, et al. The ULK1 complex mediates MTORC1 signaling to the autophagy initiation machinery via binding and phosphorylating ATG14. Autophagy 2016, 12(3):547-64.

13. Papinski D, Schuschnig M, Reiter W, Wilhelm L, et al. Early steps in autophagy depend on direct phosphorylation of Atg9 by the Atg1 kinase. Molecular cell 2014, 53(3):471-83.

14. Egan DF, Chun MG, Vamos M, Zou H, et al. Small Molecule Inhibition of the Autophagy Kinase ULK1 and Identification of ULK1 Substrates. Molecular cell 2015, 59(2):285-97.

15. Itakura E, Mizushima N. Characterization of autophagosome formation site by a hierarchical analysis of mammalian Atg proteins. Autophagy 2010, 6(6):764-76.

16. Codogno P, Mehrpour M, Proikas-Cezanne T. Canonical and non-canonical autophagy: variations on a common theme of self-eating? Nature Reviews Molecular Cell Biology 2012, 13(1):7-12.

17. Honda S, Arakawa S, Nishida Y, Yamaguchi H, et al. Ulk1-mediated Atg5-independent macroautophagy mediates elimination of mitochondria from embryonic reticulocytes. Nature Communications 2014, 5(1):4004.

18. Martinez J, Almendinger J, Oberst A, Ness R, et al. Microtubule-associated protein 1 light chain 3 alpha (LC3)-associated phagocytosis is required for the efficient clearance of dead cells. Proceedings of the National Academy of Sciences 2011, 108(42):17396.

19. Mortensen M, Soilleux EJ, Djordjevic G, Tripp R, et al. The autophagy protein Atg7 is essential for hematopoietic stem cell maintenance. The Journal of experimental medicine 2011, 208(3):455-67.

20. Mortensen M, Watson AS, Simon AK. Lack of autophagy in the hematopoietic system leads to loss of hematopoietic stem cell function and dysregulated myeloid proliferation. Autophagy 2011, 7(9):1069-70.

21. Mortensen M, Ferguson DJP, Edelmann M, Kessler B, et al. Loss of autophagy in erythroid cells leads to defective removal of mitochondria and severe anemia in vivo. Proceedings of the National Academy of Sciences 2010, 107(2):832-837.

22. Liu F, Lee JY, Wei H, Tanabe O, et al. FIP200 is required for the cell-autonomous maintenance of fetal hematopoietic stem cells. Blood 2010, 116(23):4806-14.

23. Levy JMM, Towers CG, Thorburn A. Targeting autophagy in cancer. Nature reviews Cancer 2017, 17(9):528-542. 
24. Galluzzi L, Pietrocola F, Bravo-San Pedro JM, Amaravadi RK, et al. Autophagy in malignant transformation and cancer progression. The EMBO journal 2015, 34(7):856-80.

25. Auberger P, Puissant A. Autophagy, a key mechanism of oncogenesis and resistance in leukemia. Blood 2017, 129(5):547-552.

26. Koschade SE, Brandts $\mathrm{CH}$. Selective Autophagy in Normal and Malignant Hematopoiesis. Journal of molecular biology 2020, 432(1):261-282.

27. Evangelisti C, Evangelisti C, Chiarini F, Lonetti A, et al. Autophagy in acute leukemias: a double-edged sword with important therapeutic implications. Biochimica et biophysica acta 2015, 1853(1):14-26.

28. White E, DiPaola RS. The double-edged sword of autophagy modulation in cancer. Clin Cancer Res 2009, 15(17):5308-16.

29. Ma AC, Ward AC, Liang R, Leung AY. The role of jak2a in zebrafish hematopoiesis. Blood 2007, 110(6):1824-30.

30. He BL, Shi X, Man CH, Ma AC, et al. Functions of flt3 in zebrafish hematopoiesis and its relevance to human acute myeloid leukemia. Blood 2014, 123(16):2518-29.

31. Shi X, He BL, Ma AC, Guo Y, et al. Functions of idh1 and its mutation in the regulation of developmental hematopoiesis in zebrafish. Blood 2015, 125(19):2974-84.

32. Chen XK, Kwan JS, Chang RC, Ma AC. 1-phenyl 2-thiourea (PTU) activates autophagy in zebrafish embryos. Autophagy 2020:1-10.

33. Westerfield M. The zebrafish book : a guide for the laboratory use of zebrafish (Danio rerio). 2007. English.

34. Kimmel CB, Ballard WW, Kimmel SR, Ullmann B, et al. Stages of embryonic development of the zebrafish. Dev Dyn 1995, 203(3):253-310.

35. Ma AC, Chung MI, Liang R, Leung AY. The role of survivin2 in primitive hematopoiesis during zebrafish development. Leukemia : official journal of the Leukemia Society of America, Leukemia Research Fund, UK 2009, 23(4):712-20.

36. Ma AC, McNulty MS, Poshusta TL, Campbell JM, et al. FusX: A Rapid One-Step Transcription Activator-Like Effector Assembly System for Genome Science. Human gene therapy 2016, 27(6):451-63.

37. Ma AC, Lee HB, Clark KJ, Ekker SC. High efficiency In Vivo genome engineering with a simplified 15-RVD GoldyTALEN design. PloS one 2013, 8(5):e65259.

38. Ma ACH, Chen Y, Blackburn PR, Ekker SC. TALEN-Mediated Mutagenesis and Genome Editing. Methods in molecular biology (Clifton, NJ) 2016, 1451:17-30.

39. He C, Klionsky DJ. Analyzing autophagy in zebrafish. Autophagy 2010, 6(5):642-4.

40. Sasaki T, Lian S, Qi J, Bayliss PE, et al. Aberrant autolysosomal regulation is linked to the induction of embryonic senescence: differential roles of Beclin 1 and p53 in vertebrate Spns1 deficiency. PLoS Genet 2014, 10(6):e1004409-e1004409.

41. Khuansuwan S, Barnhill LM, Cheng S, Bronstein JM. A novel transgenic zebrafish line allows for in vivo quantification of autophagic activity in neurons. Autophagy 2019, 15(8):1322-1332. 
42. Fleming A, Rubinsztein DC. Zebrafish as a model to understand autophagy and its role in neurological disease. Biochimica et Biophysica Acta (BBA) - Molecular Basis of Disease 2011, 1812(4):520-526.

43. Ma AC, Chen Y, Blackburn PR, Ekker SC. TALEN-Mediated Mutagenesis and Genome Editing. Methods in molecular biology (Clifton, NJ) 2016, 1451:17-30.

44. Lee E-J, Tournier C. The requirement of uncoordinated 51-like kinase 1 (ULK1) and ULK2 in the regulation of autophagy. Autophagy 2011, 7(7):689-695.

45. Mauthe M, Orhon I, Rocchi C, Zhou X, et al. Chloroquine inhibits autophagic flux by decreasing autophagosome-lysosome fusion. Autophagy 2018, 14(8):1435-1455.

46. Wang C, Wang H, Zhang D, Luo W, et al. Phosphorylation of ULK1 affects autophagosome fusion and links chaperone-mediated autophagy to macroautophagy. Nat Commun 2018, 9(1):3492.

47. Rožman S, Yousefi S, Oberson K, Kaufmann T, et al. The generation of neutrophils in the bone marrow is controlled by autophagy. Cell death and differentiation 2015, 22(3):445-56.

48. Lim J, Lachenmayer ML, Wu S, Liu W, et al. Proteotoxic stress induces phosphorylation of p62/SQSTM1 by ULK1 to regulate selective autophagic clearance of protein aggregates. PLoS Genet 2015, 11(2):e1004987.

49. Joo JH, Dorsey FC, Joshi A, Hennessy-Walters KM, et al. Hsp90-Cdc37 chaperone complex regulates Ulk1- and Atg13-mediated mitophagy. Molecular cell 2011, 43(4):572-85.

50. Stephenson LM, Miller BC, $\mathrm{Ng} \mathrm{A}$, Eisenberg J, et al. Identification of Atg5-dependent transcriptional changes and increases in mitochondrial mass in Atg5-deficient T lymphocytes. Autophagy 2009, 5(5):625-635.

51. Cao Y, Zhang S, Yuan N, Wang J, et al. Hierarchal Autophagic Divergence of Hematopoietic System. J Biol Chem 2015, 290(38):23050-63.

52. Lechauve C, Keith J, Khandros E, Fowler S, et al. The autophagy-activating kinase ULK1 mediates clearance of free $\alpha$-globin in $\beta$-thalassemia. Science translational medicine 2019, 11(506):eaav4881.

53. Jang JE, Eom JI, Jeung HK, Cheong JW, et al. Targeting AMPK-ULK1-mediated autophagy for combating BET inhibitor resistance in acute myeloid leukemia stem cells. Autophagy 2017, 13(4):761-762.

54. Piya S, Andreeff M, Borthakur G. Targeting autophagy to overcome chemoresistance in acute myleogenous leukemia. Autophagy 2017, 13(1):214-215.

55. Heydt Q, Larrue C, Saland E, Bertoli S, et al. Oncogenic FLT3-ITD supports autophagy via ATF4 in acute myeloid leukemia. Oncogene 2018, 37(6):787-797.

56. Liu Q, Chen L, Atkinson JM, Claxton DF, et al. Atg5-dependent autophagy contributes to the development of acute myeloid leukemia in an MLL-AF9-driven mouse model. Cell death \& disease 2016, 7(9):e2361.

57. Rudat S, Pfaus A, Cheng YY, Holtmann J, et al. RET-mediated autophagy suppression as targetable co-dependence in acute myeloid leukemia. Leukemia : official journal of the Leukemia Society of America, Leukemia Research Fund, UK 2018, 32(10):2189-2202. 
58. Watson AS, Riffelmacher T, Stranks A, Williams O, et al. Autophagy limits proliferation and glycolytic metabolism in acute myeloid leukemia. Cell Death Discov 2015, 1.

59. Jing L, Zon LI. Zebrafish as a model for normal and malignant hematopoiesis. Disease models \& mechanisms 2011, 4(4):433-8.

60. Langenau DM, Traver D, Ferrando AA, Kutok JL, et al. Myc-induced T cell leukemia in transgenic zebrafish. Science (New York, NY) 2003, 299(5608):887-90.

61. Sabaawy HE, Azuma M, Embree LJ, Tsai HJ, et al. TEL-AML1 transgenic zebrafish model of precursor B cell acute lymphoblastic leukemia. Proceedings of the National Academy of Sciences of the United States of America 2006, 103(41):15166-71.

62. Ma AC, Fan A, Ward AC, Liongue C, et al. A novel zebrafish jak2a(V581F) model shared features of human JAK2(V617F) polycythemia vera. Experimental hematology 2009, 37(12):1379-1386.e4.

63. Xu M, Ye Y, Ye Z, Xu S, et al. Human BCR/ABL1 induces chronic myeloid leukemia-like disease in zebrafish. Haematologica 2020, 105(3):674-686.

64. He C, Bartholomew CR, Zhou W, Klionsky DJ. Assaying autophagic activity in transgenic GFP-Lc3 and GFP-Gabarap zebrafish embryos. Autophagy 2009, 5(4):520-6.

65. Raby L, Volkel P, Le Bourhis X, Angrand PO. Genetic Engineering of Zebrafish in Cancer Research. Cancers (Basel) 2020, 12(8). 


\section{Figure Legends}

Figure 1. Loss of $u l k 1 b$ inhibits autophagy activation but not autophagic flux in zebrafish. (A) Imaging of $\mathrm{Lc}^{+}$cells in the midbrain. The relative number of autophagosome (GFP- $L c 3$ ), lysosome (LysoTracker-red ${ }^{+}$) and autolysosome (merged-white ${ }^{+}$, fusion of GFP-Lc3 and LysoTracker-red ${ }^{+}$) puncta per cell in the midbrain neuron were counted based on Z-Stack image analysis (20 layers out of 100 layers) with maximal intensity projection (MIP). All four groups $\left(u l k 1 b^{+/+}\right.$in $\mathrm{E} 3, u l k 1 b^{-/-}$in $\mathrm{E} 3, u l k 1 b^{+/+}$in CQ and $u l k 1 b^{-/-}$in CQ) individually comprises in a total of at least ten $\operatorname{Tg}(\mathrm{GFP}-L c 3)$ experimental embryos to complete three biological replicates. Red and yellow boxes indicating the autophagosome (GFP-Lc3) and autolysosome (merged-white ${ }^{+}$, GFPLc3 and LysoTracker-red+) puncta respectively. Representative bar diagrams of showing the number of co-localized puncta in the neuron cell of $4 \mathrm{dpf}$ drug treated and untreated zebrafish embryos. Scale bar: $40 \mu \mathrm{m}$ (Merged) and $4 \mu \mathrm{m}$ (Enlarged). (B) Western blot results showing the Lc3-II protein level of wild type siblings $\left(u l k 1 b^{+/+}\right)$and homozygous mutants $\left(u l k 1 b^{-/}\right)$treated with $100 \mu \mathrm{M}$ CQ. Relative Lc3-II protein level was normalized by GAPDH while set up the $u l k 1 b^{+/+}$value as 1.0. Each group comprising in a total of 75 embryos for three independent experiments. Blue color box: $u l k 1 b^{+/+}$in E3; red color box: $u l k 1 b^{-/-}$in E3; black color box: $u l k 1 b^{+/+}$ in CQ; brick red color box: $u l k 1 b^{-/-}$in CQ. (C) Representative images showing the myeloid cell specific autophagy in between $3 \mathrm{dpf}$ double transgenic [Tg(GFP-Lc3;corola:mCherry)] homozygous siblings and mutant zebrafish embryos treated with E3 fish water. Scale bar: $5 \mu \mathrm{m}$. In Panel (A) and (B), statistical analysis were performed by two-way analysis of variance (ANOVA) using Tukey's post-hoc method and error bars were presented here as mean \pm standard error of mean (SEM). ${ }^{*} P<0.05,{ }^{*} * P<0.01, * * * P<0.001, * * * * P<0.0001, \# \# P<0.01$ compared to the $u l k 1 b^{-}$ ${ }^{-}$mutants and ns: non-significant.

Figure 2. Autophagy deficient zebrafish larvae showed aberrant hematopoietic phenotypes during myelopoiesis. (A) In definitive hematapoiesis, ulklb deficient homozygous mutants presented upregulation in spilb, lcpl and mpx at $2 \mathrm{dpf}$ in the CHT region compared to the wild type siblings $\left(u l k 1 b^{+++}\right)$whereas $m y b$ and hbael.1 expressions were remarkably decreased at similar time points. (B) Similarly, in the ulk2 mutants, spilb, lcpl and mpx expressions in the CHT region significantly upregulated compared with the wild type siblings whereas myb and hbael.1 decreased. Scale bar: $300 \mu \mathrm{m}$. In Panel (A) and (B), statistical analysis were performed by MannWhitney U test. All the whiskers, boxes, and central lines were shown as minimum-to-maximum values, 25th-to-75th percentile, and the 50th percentile (median), respectively. $* P<0.05, * * P<0.01$, $* * * P<0.001$.

Figure 3. 3-MA perturbed autophagosome formation and myelopoiesis. (A) Schematic illustration showing the imaging of $\mathrm{Lc}^{+}$cells in the midbrain section. The relative number of autophagosome (GFP-Lc3), lysosome (LysoTracker-red ${ }^{+}$) and autolysosome (merged-white ${ }^{+}$, GFP-Lc3 and LysoTracker-red ${ }^{+}$) puncta per cell in the midbrain neuron were counted based on Zstack image analysis (20 layers out of 100 layers) with maximal intensity projection (MIP). Both 3-MA treated and untreated groups individually comprises in a total of at least ten $\mathrm{Tg}(\mathrm{GFP}-L c 3)$ 
experimental embryos to complete three biological replicates. Yellow and red dash boxes showing the autophagosome (GFP-Lc3) and autolysosome (merged-white ${ }^{+}$, GFP-Lc3 and LysoTracker$\mathrm{red}^{+}$) puncta respectively. 3-MA: 3-Methyladenine. Representative bar diagrams of showing the number of co-localized puncta in the neuron of $4 \mathrm{dpf} 3-\mathrm{MA}$ treated and untreated zebrafish embryos in the midbrain section. (B) In situ hybridization indicated that treatment with early autophagy inhibitor 3-MA significantly disrupted definitive hematopoiesis in the control siblings including aberrant expression of $l c p l$ and $m p x$ as well as ectopic downregulation of $m y b$ and hbae1.1 in the CHT. hpf: hours post fertilization, dpf: days post fertilization, Scale bar: $300 \mu \mathrm{m}$. Scale bar: $40 \mu \mathrm{m}$ (Merged) and $4 \mu \mathrm{m}$ (Enlarged). In Panel (A) and (B), statistical analysis were performed by Mann-Whitney U test. In panel (A), error bars were presented here as mean \pm standard error of mean (SEM) and in panel (B), all the whiskers, boxes, and central lines were shown as minimum-to-maximum values, $25^{\text {th }}$-to- $75^{\text {th }}$ percentile, and the $50^{\text {th }}$ percentile (median), respectively. $* P<0.05, * * P<0.01$ and $\mathrm{ns}$, non-significant.

Figure 4. Calpeptin can partially recapitulate autophagy and ameliorate defective hematopoiesis in zebrafish mutants. (A) Schematic illustration showing the imaging of $\mathrm{Lc}^{+}$ cells in the midbrain section. The relative number of autophagosome (GFP-Lc3), lysosome $\left(\right.$ Lyso Tracker-red $^{+}$) and autolysosome (merged-white ${ }^{+}$, fusion of GFP-Lc3 ${ }^{+}$and LysoTracker-red ${ }^{+}$) puncta per cell in the midbrain neuron were counted based on Z-Stack image analysis (20 layers out of 100 layers) with maximal intensity projection (MIP). All four groups $\left(u l k 1 b^{+/+}\right.$in DMSO, $u l k 1 b^{-/}$in DMSO, $u l k 1 b^{+/+}$in calpeptin and $u l k 1 b^{-/-}$in calpeptin) individually comprises in a total of at least ten $\mathrm{Tg}(\mathrm{GFP}-\mathrm{Lc} 3)$ experimental embryos to complete three biological replicates. Yellow and red color boxes indicating the autophagosome $\left(\right.$ GFP-Lc3 ${ }^{+}$) and autolysosome (merged-white ${ }^{+}$) puncta respectively. Representative bar diagrams of showing the number of co-localized autophagosome, lysosome and autolysosome puncta in the neuron cell of $4 \mathrm{dpf}$ calpeptin treated and untreated zebrafish embryos. Scale bar: $40 \mu \mathrm{m}$ (Merged) and $4 \mu \mathrm{m}$ (Enlarged). (B) In situ hybridization showed that calpeptin can partially rescue the $u l k 1 b$ mutant's $l c p 1, m p x, m y b$ and hbael.1 phenotypes compared to the wild type siblings at 2 dpf. Scale bar: $300 \mu \mathrm{m}$. In panel (A), statistical analysis was performed by two-way analysis of variance (ANOVA) using Tukey's posthoc method and error bars were presented here as mean \pm standard error of mean (SEM). In panel (B), the whiskers, boxes, and central lines were shown as minimum-to-maximum values, $25^{\text {th }}$-to$75^{\text {th }}$ percentile, and the $50^{\text {th }}$ percentile (median), respectively. $* P<0.05, * * P<0.01$ and $* * * P<0.001$ and ns, non-significant. 


\section{Figures}

Figure 1.
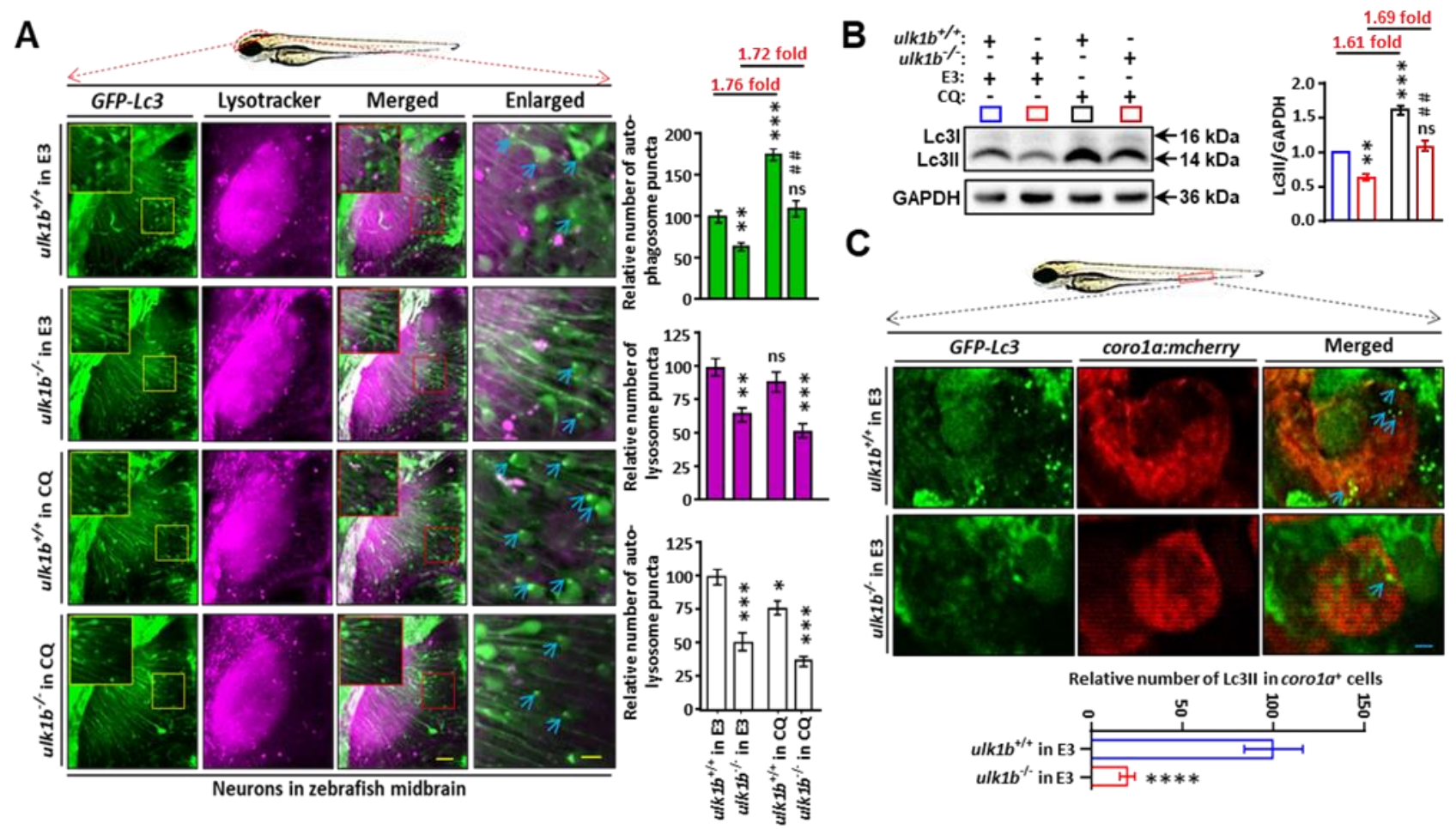
bioRxiv preprint doi: https://doi.org/10.1101/2021.06.14.448302; this version posted June 14, 2021. The copyright holder for this preprint (which was not certified by peer review) is the author/funder, who has granted bioRxiv a license to display the preprint in perpetuity. It is made available under aCC-BY-NC-ND 4.0 International license.

Figure 2.

A
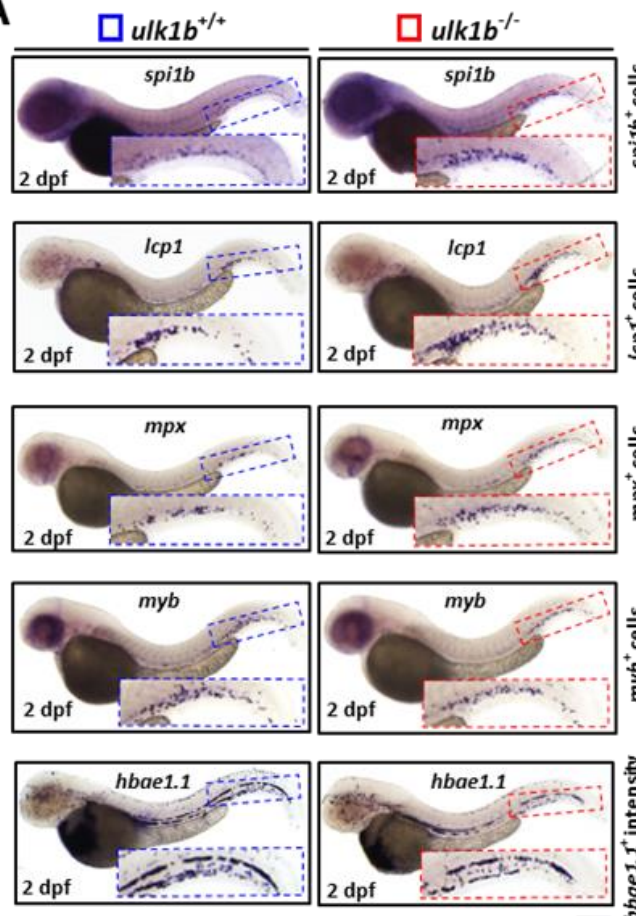
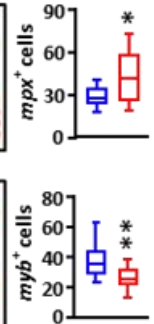

B
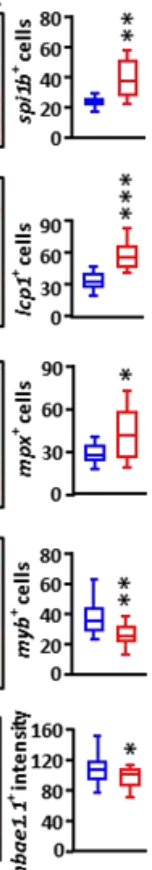
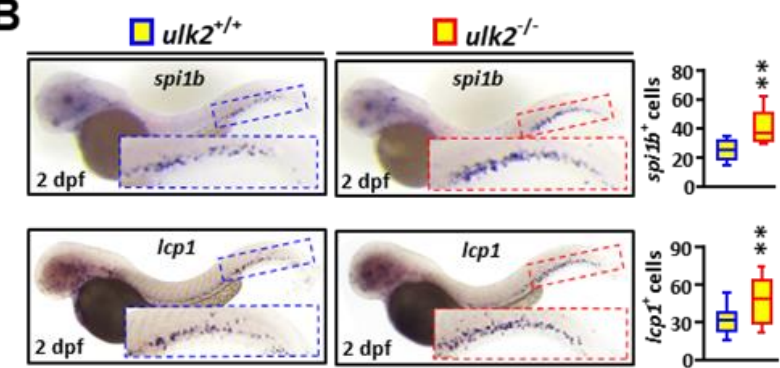

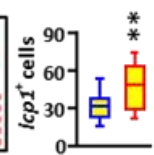
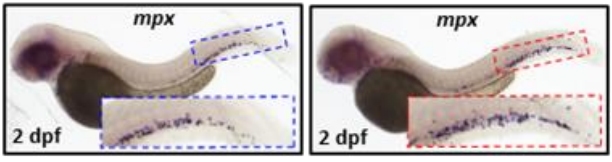

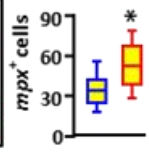
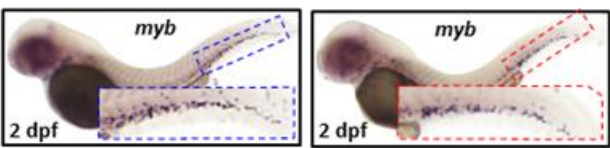

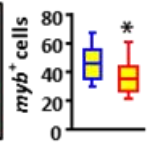
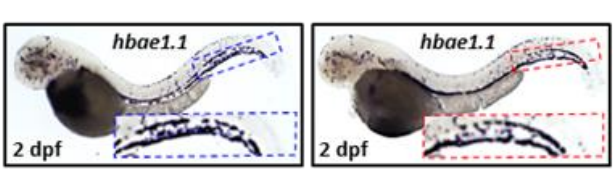

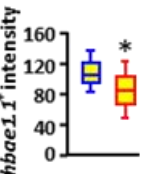

Figure 3.

A
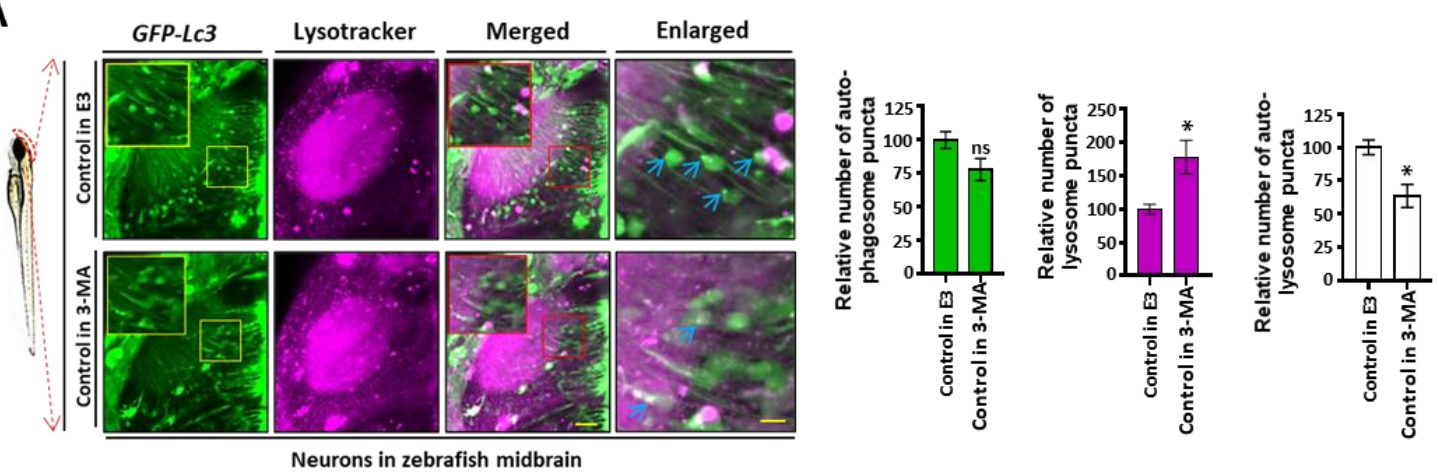

B
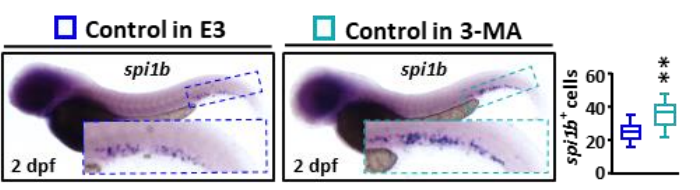

$\square$ Control in E3

$\square$ Control in 3-MA
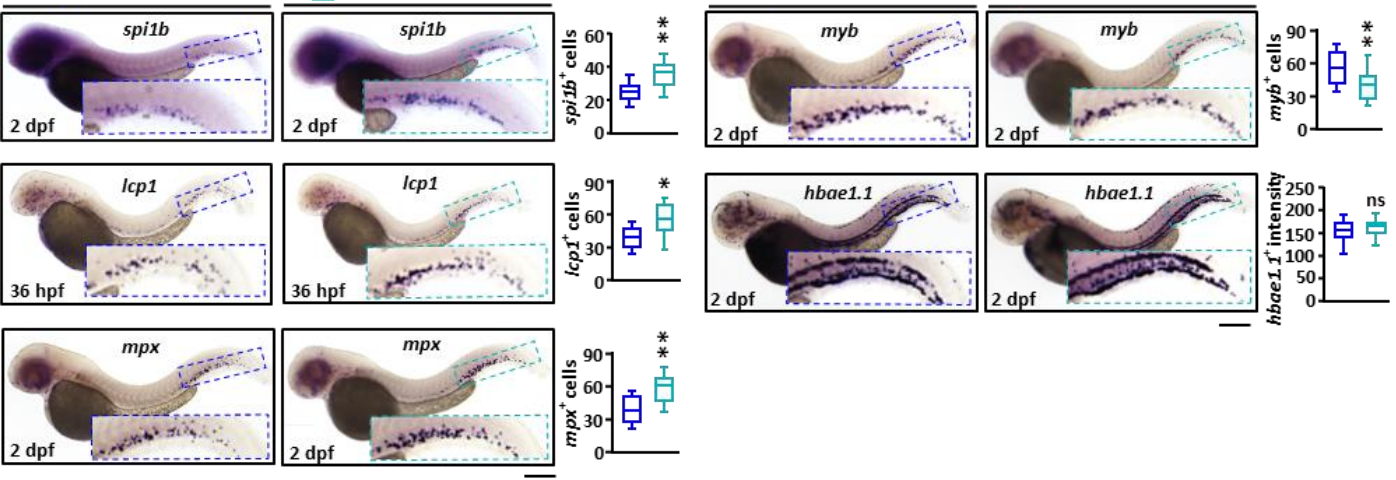
bioRxiv preprint doi: https://doi.org/10.1101/2021.06.14.448302; this version posted June 14, 2021. The copyright holder for this preprint (which was not certified by peer review) is the author/funder, who has granted bioRxiv a license to display the preprint in perpetuity. It is made available under aCC-BY-NC-ND 4.0 International license.

Figure 4.

A

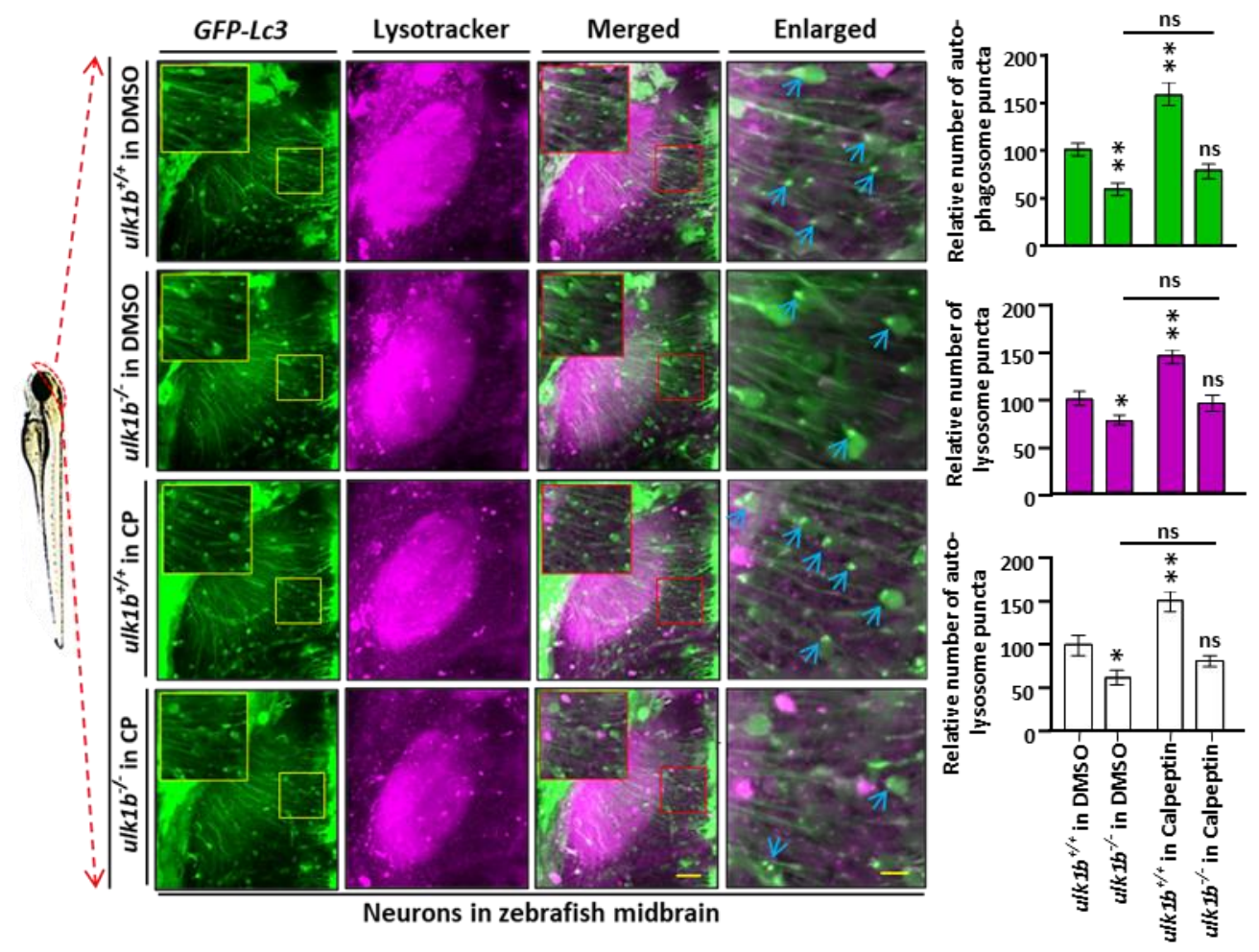

B
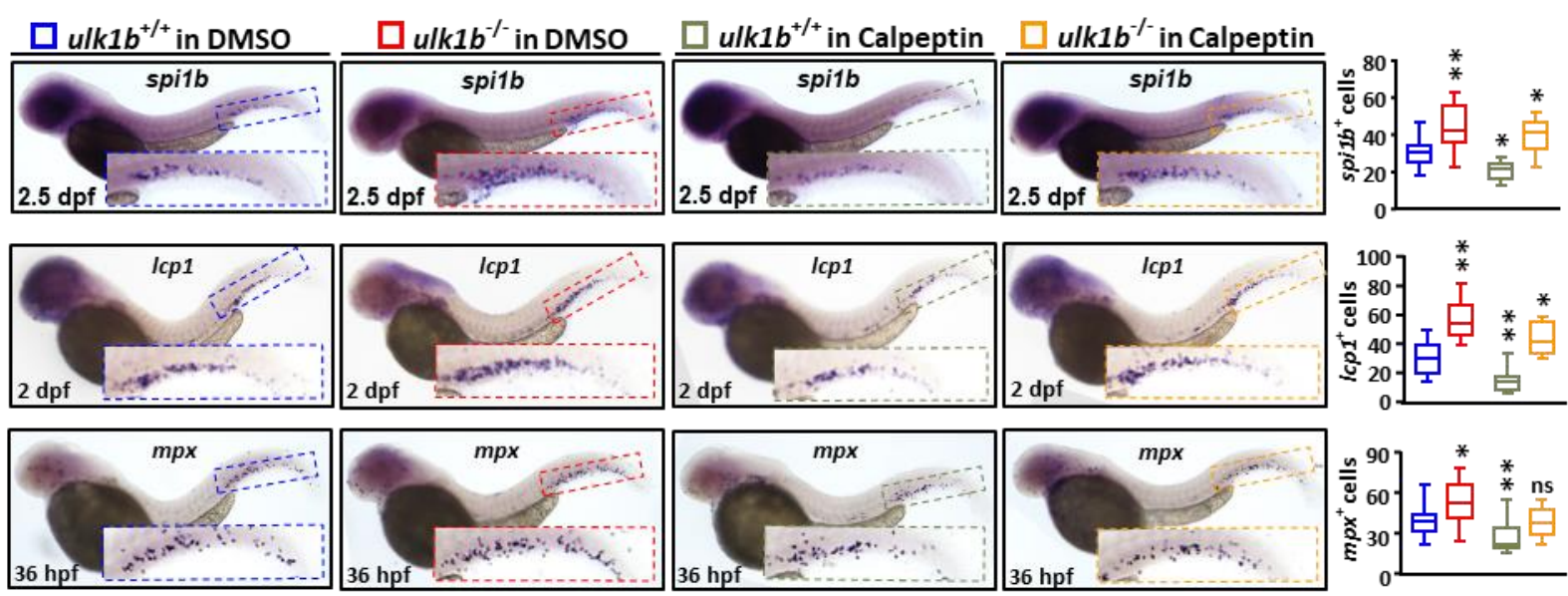

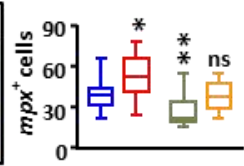
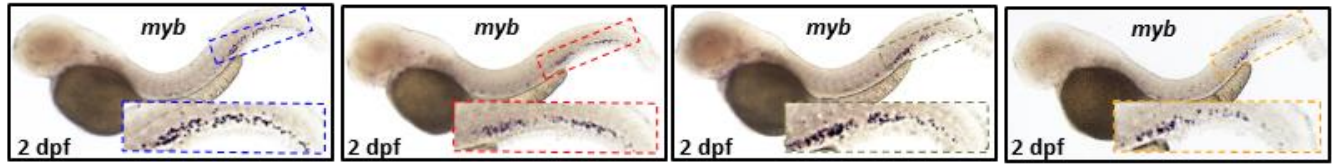

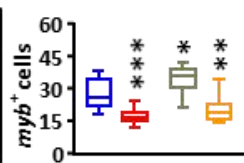
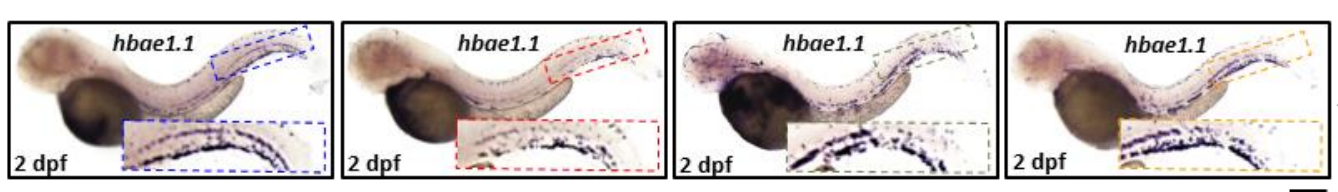

胥200 